\title{
GROUND-BASED PHOTOMETRIC CALIBRATION OF THE SPACE TELESCOPE CCD CAMERA
}

\author{
D.A. Hunter ${ }^{1}$, H.C. Harris ${ }^{2}$, W.A. Baum ${ }^{1}$, J.H. Jones ${ }^{1}$, T.J. Ireidl ${ }^{1}$ \\ 1 Lowell Observatory, 1400 West Mars Hill Rd., Flagstaff, AZ U.S.A. \\ 2 U.S. Naval Observatory, PO Box 1149, Flagstaff, AZ U.S.A.
}

\section{Introduction}

The Wide-Field and Planetary Camera (WF/PC) is a CCD imaging instrument which is part of the Hubble Space Telescope. Ground-based observations have been made with a CCD system similar to those of the WF/PC in order to establish the standard star sequence to be used for in-flight photometric calibration. Because the WF/PC passbands differ from those in previous photometric use, the filters and CCDs will define a new photometric system. We outline here the procedures used to establish the calibration fields to be used in flight (see Harris et al. 1988 for additional details).

Two globular cluster fields were chosen for use as calibrators: a position 13.6' southwest of the center in $\omega$ Cen and a position 10.2' northwest of the center in NGC 6752. Observations were made with copies of 15 of the $42 \mathrm{WF} / \mathrm{PC}$ filters, covering 3000 to $10000 \AA$ (see Griffiths 1985 for filter curves). Five of these filters approximate the Johnson-Cousins UBVRI system. However, the filters that are likely to be the most popular substitutes for $V$ and $I-F 555 \mathrm{~W}$ and F785LP - are broader than the Johnson-Cousins filters.

\section{Observations}

The observations were made using the Las Campanas 1-m telescope in 1985. Additional observations were made at Lowell Observatory. The ground-based instrument was a thinned TI $816 \times 800$-pixel CCD. The chip was coated with coronene so that ultraviolet photons below $3800 \AA$ cause the chip to fluoresce in the green where the quantum efficiency of the chip is higher (Blouke et al. 1980). To improve the quantum efficiency and stability, the uncooled chip was flooded with ultraviolet light by illuminating the window with a mercury discharge lamp for 30-60 minutes during pumping prior to observing.

Most of the images were flat-fielded using exposures of a white spot projected on the dome. A color-balancing filter was used to approximate the color of the night sky, and for the widest passbands this made a difference of $2 \%$ in the flat fields. For F336W twilight sky flats were used because of the greater photon flux. Potential problems with using twilight sky as flats include the presence of emission lines, polarization, and the blue color. Tests indicated, however, that these problems contribute errors less than $1 \%$. The instrument was sufficiently stable that an average flat could be constructed for a given observing run. 
Approximately 30 Landolt $(1973,1983,1987)$ standard stars were observerl each night, and stars were observed several times at different locations on the detector and at different air masses. For a few bright red stars the telescope was defocussed to keep the exposure time over 3 seconds. The cluster exposures consisted of a short- and long-integration pair, typically 1 and 5 minutes, and were repeated on $2-5$ nights. Approximately 2000 CCD frames were obtained.

\section{Standard star reductions}

Aperture photometry was simulated using DAOPHOT (Stetson 1987) to determine the magnitudes of the standard stars. Growth curves were determined, and the magnitudes were corrected to a common 25 " aperture. The correction depended on the seeing and was typically $1-2 \%$. An iterative least squares fit was used to place each night on a common internal system. A color term was unnecessary. The mean sigma in the fits was $0.003-0.018$ magnitude. The filters $\mathrm{F} 336 \mathrm{~W}$ and $\mathrm{F} 1042 \mathrm{M}$ gave worse photometric errors at all steps of the process. For F336W the sigma in the fit is 0.04 and for F1042M it is 0.03 magnitude.

Atmospheric extinction determinations were modelled using Tüg et al.'s (197T) extinction as a function of wavelength and extrapolated to $2900 \AA$ (Allen 1973). This formulation was applied for air masses of 1.2 and 2.0 to the Gunn and Stryker (1983) stellar spectral atlas, WF/PC passband magnitudes were synthesized, and extinction coefficients were calculated. A color term was necessary and (F555W - F785LP) was used. For F336W a quadratic term in air mass was also required.

The final zero point of the system was defined so that an $A O V$ star has colors of zero. To accomplish this a plot was made of the WF/PC magnitude minus the nearest UBVRI magnitude versus the UBVRI color index. The zero point is where the fit of these points intersects the origin. However, the curvature of the fit was determined not from the standarrl stars, which were too few, but from magnitudes synthesized from the stellar spectra of Gunn and Stryker (1983). A least squares fit was then applied to the standard stars with the coefficient of the quadratic color term being set by the Gunn and Stryker stars. Only normal giants and dwarfs were included. Sigmas of the fits were $0.007-0.037$ with an average of 0.02 magnitude, excluding F336W and F1042M.

\section{The cluster field reductions}

Because the cluster fields are crowded, DAOPHOT was used to measure the stellar photometry. A master coordinate list was set up for each cluster using one of the deep images and was transformed to the coordinate system of subsequent images. Only a few frames were deeper than the one used to set up the master list of stars. Stars over most of the field of view of the original images were included: about 3100 stars in $\omega$ Cen and about 1300 stars in NGC 6752. The 91 cluster frames were reduced on the WF/PC-team Micro Vax II workstation and the SSC Cray X-MP/48 with time provided through NOAO and code provided by M. Mateo. Approximately $10 \%$ of the stars were deleted on the basis of quality indicators returned by DAOPHOT.

After the magnitudes were obtained with the NSTAR fitting routine, the aperture correction was determined using the short exposure of the short-long pair. The long exposure was forced to agree with the short exposure as determined by stars that were well measured on both frames. The cluster magnitudes were placed on the same aperture system as the standard stars using the growth curves of standard stars bracketting the cluster in time. 
The cluster magnitudes were corrected for extinction in the same manner as for the standard stars. Observations made on different nights were compared. If there was any systematic offset, the difference was split and added or subtracted as a constant to each of the nights since there could be stars that were measured on one frame and not on another. Typical offsets were less than 0.02 magnitude. The results from different frames were combined and the zero point constant added. Magnitudes were weighted by sigma. A sigma was computed that is a combination of the sigmas of individual observations and one that is the dispersion around the mean. The higher of the two sigmas was adopted as the final measure of uncertainty. Figure 1 shows the final sigmas of all of the stars which were measured in $\omega$ (en as a function of the magnitude for filter F555W. Figure 2 shows a F569W vs. F569W - F 791W ( V vs. $V-I$ ) color-magnitude diagram.

\section{$5 \quad$ Selection of standard stars}

Bright, relatively unblended stars of low sigma in the WF/PC fields were selected as stars to be used as in-flight standards. In addition some fainter stars with somewhat higher sigmas were included in the center of the fields in order to increase the number of stars available to the limited field of view of the Planetary Camera. In $\omega$ Cen 80 stars and in NGC 675267 stars were selected. The standard stars have sigmas which range from 0.005 to 0.03 with an average of about 0.01 (see Figure 3 ).

\section{References}

Allen, C.W. 1973, Astrophysical Quantities, (London: Athlone).

Blouke, M.M., Crowens, M.W., Hall, J.E., Westphal, J.A., and Christensen, A.B. 1980, Applied Optics, 19, 3318.

Griffiths, R. 1985, Wide Field and Planetary Camera Instrument Handbook (STScI).

Gunn, J.E. and Stryker, L.L. 1983, Ap J Suppl, 52, 121.

Harris, H.C., Hunter, D.A., Baum, W.A., Jones, J.H., and Kreidl, T.J., in preparation.

Landolt, A.U. 1973, A J,78, 959.

-1983, A J 88,439 .

- 1987, Letter to STScI.

Stetson, P.B. 1987, P A S P,99, 191.

Tüg, H., White, N.W., Lockwood, G.W. 197т, A A.61, 679. 

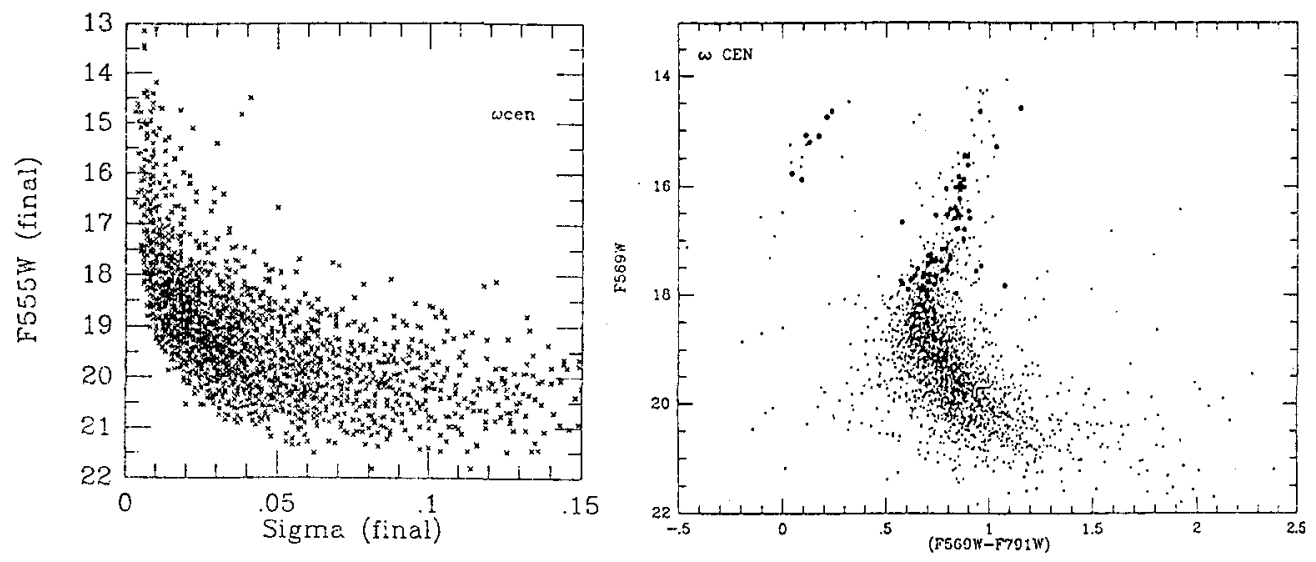

Fig. 1-The uncertainty in the magnitudes of $\mathrm{F} 555 \mathrm{~W}$ are shown as a function of magnitude for all of the stars measured in the $\omega$ Cen field.

Fig. 2-A color-magnitude diagram ( $\sim V-I$ vs. V) for the stars measured in the $\omega$ Cen field. The enlarged dots are stars chosen for use as in-flight standards.

Fig. 3-The uncertainty in the F555W magnitudes are shown for the standard stars chosen in the $\omega$ Cen field.

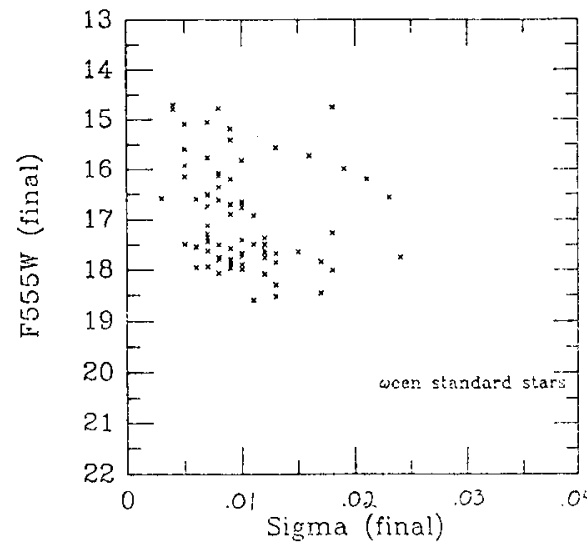

